\title{
Samambaias e licófitas do Rancho Souza, município de Abaetetuba, estado do Pará,
} Brasil

\author{
Ferns and lycophytes from Rancho Souza, municipality of Abaetetuba, Pará State, Brazil \\ Helechos y licofitas del Rancho Souza, municipio de Abaetetuba, estado de Pará, Brasil
}

Recebido: 17/12/2021 | Revisado: 21/01/2022 | Aceito: 29/01/2022 | Publicado: 30/01/2022

\author{
Jeferson Miranda Costa \\ ORCID: https://orcid.org/0000-0001-7896-7703 \\ Instituto Federal de Educação, Ciência e Tecnologia do Pará, Brasil \\ E-mail: jeferson.costa@ifpa.edu.br \\ Suellen Bonneterre Pereira Rodrigues \\ ORCID: https://orcid.org/0000-0003-0784-1104 \\ Instituto Federal de Educação, Ciência e Tecnologia do Pará, Brasil \\ E-mail: suellenbonneterre@ hotmail.com \\ Luís Paulo Silva da Cunha \\ ORCID: https://orcid.org/0000-0003-3699-2075 \\ Instituto Federal de Educação, Ciência e Tecnologia do Pará, Brasil \\ E-mail: luis.bio.cunha@gmail.com
}

\begin{abstract}
Resumo
Este trabalho apresenta um levantamento florístico das samambaias e licófitas do Rancho Souza, município de Abaetetuba, estado do Pará, Brasil, com chave para identificação das espécies e informações sobre seus respectivos hábitos, formas de vida, ambientes de ocorrência e padrões de distribuição geográfica. Na área de estudo, foram registradas 31 espécies de plantas vasculares sem sementes, sendo uma licófita (Selaginella conduplicata Spring, Selaginellaceae) e 30 samambaias, distribuídas em 22 gêneros e 14 famílias. As famílias mais representativas foram Pteridaceae (6 spp.), Hymenophyllaceae (4 spp.) e Polypodiaceae (4 spp.). Os gêneros com maior riqueza específica foram Cyathea J. Sm. (3 spp.) e Didymoglossum Desv. (3 spp.). A maioria das espécies tem hábito herbáceo (26 spp.), formas de vida terrestres (17 spp.) e epífitas (12 spp.), distribuição americana ( $25 \mathrm{spp}$.), e ocorre no interior da mata (24 spp.). Estes resultados demonstraram a importância da preservação dos remanescentes florestais em áreas sob forte pressão antrópica para a conservação da flora regional de samambaias e licófitas.
\end{abstract}

Palavras-chave: Amazônia brasileira; Florística; Plantas vasculares sem sementes.

\begin{abstract}
This work presents a floristic survey of the ferns and lycophytes from Rancho Souza, municipality of Abaetetuba, Pará State, Brazil, with identification key to species and information on their habit, life form, occurring environment and pattern of geographical distribution. In the study area, 31 species of seedless vascular plants were recorded, being one lycophyte (Selaginella conduplicata Spring, Selaginellaceae) and 30 ferns, distributed in 22 genera and 14 families. The most representative families were Pteridaceae (6 spp.), Hymenophyllaceae (4 spp.) and Polypodiaceae (4 spp.). The genera with highest species richness were Cyathea J. Sm. (3 spp.) e Didymoglossum Desv. (3 spp.). The majority of the species have herbaceous habit (26 spp.), terrestrial (17 spp.) and epiphytic (12 spp.) life forms, American distribution (25 spp.), and inhabit the forest interior (24 spp.). These results demonstrate the importance of preserving forest remnants in areas under strong anthropic pressure for the conservation of regional ferns and lycophytes flora.
\end{abstract}

Keywords: Brazilian Amazon; Floristic; Seedless vascular plants.

\section{Resumen}

Este trabajo presenta un relevamiento florístico de helechos y licofitas de Rancho Souza, municipio de Abaetetuba, estado de Pará, Brasil, con claves para la identificación de especies e información sobre sus respectivos hábitos, formas de vida, ambientes de ocurrencia y patrones de distribución geográfica. En el área de estudio se registraron 31 especies de plantas vasculares sin semillas, una licofita (Selaginella conduplicata Spring, Selaginellaceae) y 30 helechos, distribuidos en 22 géneros y 14 familias. Las familias más representativas fueron Pteridaceae (6 spp.), Hymenophyllaceae (4 spp.) y Polypodiaceae (4 spp.). Los géneros más diversos fueron Cyathea J. Sm. (3 spp.) y Didymoglossum Desv. (3 spp.). La mayoría de las especies tienen hábitos herbáceos (26 spp.), formas de vida terrestres (17 spp.) y epífitas (12 spp.), distribución americana (25 spp.), y se encuentran en el interior del bosque (24 spp.). Estos resultados demuestran la importancia de preservar los remanentes de bosques en áreas bajo fuerte presión antropogénica para la conservación de la flora regional de helechos y licofitas.

Palabras clave: Amazonia brasileña; Florística; Plantas vasculares sin semillas. 


\section{Introdução}

A causa mais comum da atual extinção global de espécies continua a ser a destruição e alteração do habitat natural, em particular, os desmatamentos nas regiões tropicais, onde geralmente samambaias e licófitas constituem uma porção significativa da flora vascular, motivo pelo qual são consideradas extremamente vulneráveis à extinção (Arcand \& Ranker, 2008). No Brasil, por exemplo, as pteridófitas representam cerca de 4,5\% das plantas vasculares registradas na floresta amazônica (Flora do Brasil 2020), cuja área desmatada atingiu $813.485 \mathrm{Km}^{2}$ em 2020, representando 20,35\% de área florestal da Amazônia Legal, segundo dados do Instituto Nacional de Pesquisas Espaciais (INPE, 2020).

É importante frisar que a taxa de desflorestamento é mais intensa ao longo das bordas sul e leste da Amazônia, na região conhecida como "arco do desmatamento" (Fearnside, 2020), com a perda de biodiversidade sendo a principal conseqüência (Vieira et al., 2005). Isso porque a maioria das espécies de samambaias, licófitas e outros seres que habitam o interior de florestas maduras não é capaz de crescer em outros tipos de ambientes, como pastos, plantações e florestas secundárias (Zuquim et al., 2008). No arco do desmatamento da Amazônia, a região nordeste do Pará é reconhecida por ser uma das áreas críticas para as espécies ameaçadas de extinção (Coelho et al., 2018), uma vez que a maior parte de sua vegetação original já foi devastada ou fortemente alterada (Cordeiro et al., 2017).

Contudo, o nordeste do Pará ainda apresenta remanescentes florestais relativamente bem preservados ou em diversos estágios de regeneração (matas secundárias), geralmente representados por fragmentos florestais associados a nascentes e cursos d'água (matas ciliares) que se encontram imersos em matrizes de ambiente degradado. É o caso do Rancho Souza, localizado no munícipio de Abaetetuba-PA, onde os proprietários buscam preservar um remanescente florestal, que inclui áreas de terra firme e várzea com condições favoráveis à ocorrência de samambaias e licófitas da flora nativa da região.

Desta forma, o presente estudo teve como objetivos realizar um levantamento das samambaias e licófitas ocorrentes no Rancho Souza, município de Abaetetuba (Pará, Brasil), bem como apresentar uma chave de identificação para as espécies e informações sobre seus hábitos, forma de vidas, ambientes de ocorrência e padrões de distribuição.

\section{Material e Métodos}

\subsection{Localização e caracterização da área de estudo}

O Rancho Souza está localizado na zona rural do município de Abaetetuba, estado do Pará, Brasil, entre as

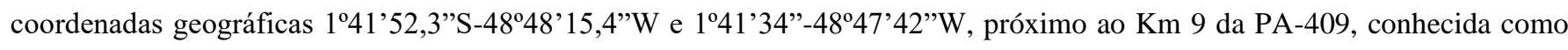
"estrada de Beja" por ligar a sede municipal ao distrito de Beja (Figura 1). 
Figura 1. Mapa de localização da área de estudo: A. Localização do município de Abaetetuba (círculo) no estado do Pará. B. Localização do Rancho Souza (círculo) no município de Abaetetuba. C. Delimitação do Rancho Souza.
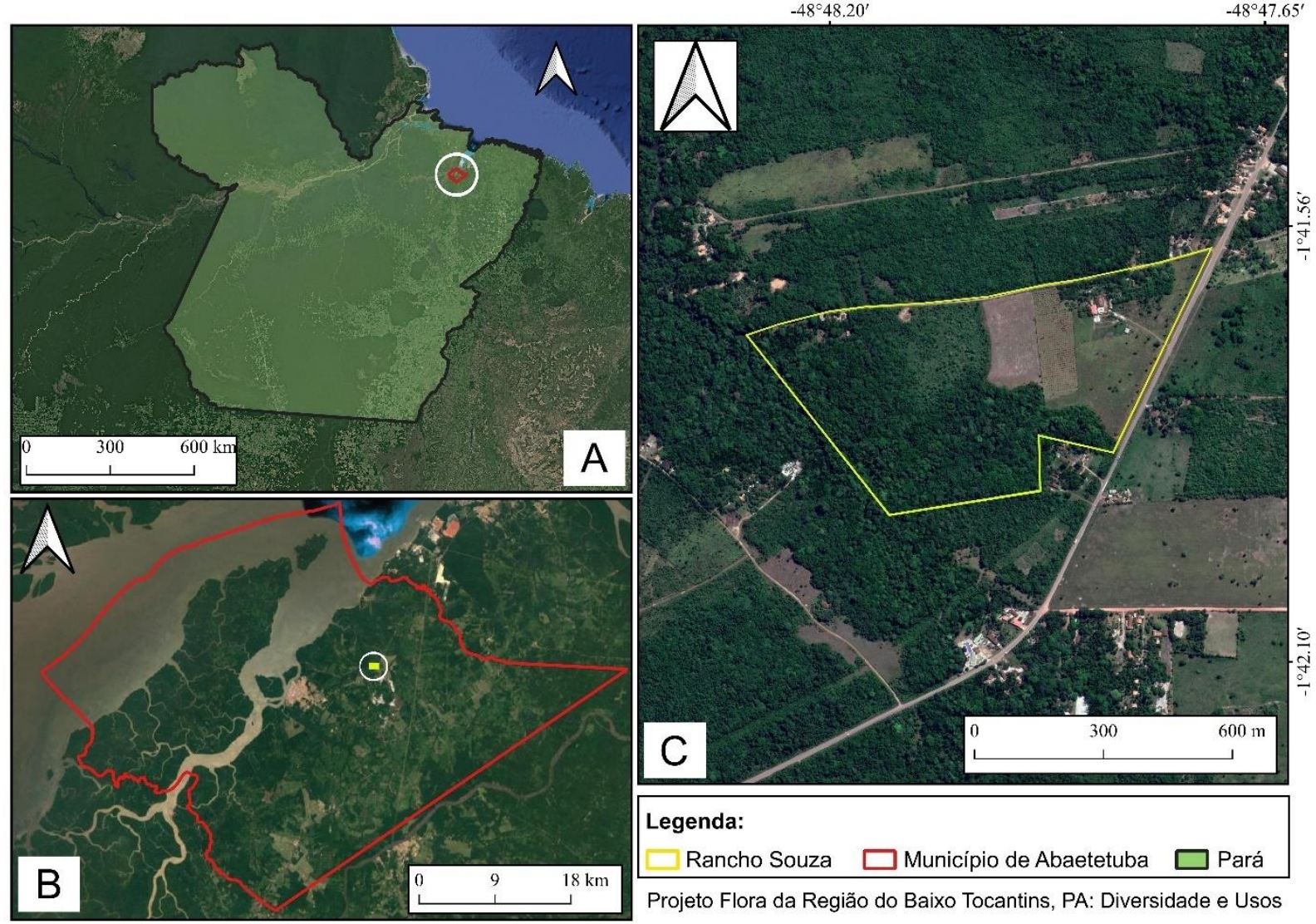

Fonte: Arquivos do HIFPA (elaborado por Ricardo Leite Ferreira Filho, com modificações de Jeferson Miranda Costa).

Trata-se de uma propriedade particular com cerca de 50 hectares que compreende parte do remanescente florestal associado a um afluente do rio Guajará de Beja, conhecido como "Igarapé do Batismo". Este trecho de mata encontra-se em regeneração natural e apresenta áreas de terra firme e várzea (Figura 2), sendo delimitado por ramais, avenida (PA-409) e áreas de pasto, roçado, plantio e residências.

O clima da área é do tipo Am segundo a classificação de Köppen, correspondendo à categoria de super úmido, apresentando altas temperaturas, inexpressiva amplitude térmica e precipitações ambulantes (FAPESPA, 2015).

\subsection{Levantamento e identificação das espécies}

Para o levantamento das espécies, consultou-se a coleção de samambaias e licófitas do Herbário do Instituto Federal do Pará - Campus Abaetetuba (HIFPA), onde estão depositadas as amostras coletadas no Rancho Souza durante quatro excursões feitas nos meses de setembro de 2010, fevereiro, maio e agosto de 2011.

A identificação das espécies foi feita com base em revisões taxonômicas, monografias e floras publicadas para diferentes países e regiões dos neotrópicos, sobretudo Boer (1962), Tryon e Tryon (1982), Moran (1987, 2000), Moran e Riba (1995), Berry et al. (1995), Mickel e Smith (2004), Prado e Moran (2008), Christenhusz (2010), Weigand e Lehnert (2016), Cárdenas et al. (2019), Fernandes e Salino (2020) e as monografias do projeto Flora do Brasil 2020. 


\subsection{Tratamento florístico e taxonômico}

A circunscrição adotada para as famílias e gêneros de samambaias e licófitas segue o sistema de classificação proposto pelo PPG I (2016). Os nomes aceitos das espécies e a abreviação dos nomes dos autores dos táxons estão de acordo com a Flora do Brasil 2020.

Os aspectos ecológicos das espécies foram determinados a partir das observações de campo presentes nas etiquetas das exsicatas analisadas e nas fotos de coleta (Figuras 2, 3 e 4) presentes no banco de imagens do HIFPA, sendo categorizados e definidos a seguir:

Quanto ao hábito, as espécies foram classificadas em: Herbáceas (HE) - Plantas em geral de pequeno porte com caule carecendo ou possuindo pouco tecido lenhoso (Mori et al., 1989); Arborescentes (AR) - Plantas com caules simples, lenhosos, constituídos apenas por tecidos primários, podendo atingir vários metros de altura e com uma roseta de grandes folhas no ápice (Fernandes, 2003); Trepadeiras (TR) - Plantas com frondes escandentes de raque volúvel (Ferreira et al., 2009; Pietrobom et al., 2015).

As formas de vida das espécies ocorrentes no Rancho Souza foram classificadas, conforme as definições de Zuquim et al. (2008), nas seguintes categorias: Terrestres (TE) - Plantas que nascem e passam todo o ciclo de vida em contato com o solo; Epífitas (EP) - Plantas que nunca entram em contato direto com o solo, pois nascem em tronco de árvores ou palmeiras nutrindo-se da água da chuva que escorre por folhas e galhos; Hemiepífitas (HE) - Plantas que nascem no solo e, posteriormente, crescem sobre outras plantas, reproduzindo-se apenas depois de atingirem determinada altura.

Os ambientes de ocorrência identificados na área de estudo foram: Interior da Mata (IM) - Ambiente em geral úmido e sombreado localizado no interior do remanescente florestal (Figura 2A,C); Clareira (CL) - Área aberta na floresta pela remoção ou queda natural de árvores de grande porte (Figura 2B); Margem da Mata (MM) - Ambiente de contato entre a floresta e a matriz de ambiente degradado que a envolve (Figura 2D); Local Aberto (LA) - Ambientes não associados ao remanescente florestal, como áreas de pasto, roçado, plantio e residências (Figura 2E,F).

Os padrões de distribuição geográfica das espécies seguem as definições de Moran e Smith (2001) e Parris (2001), com modificações propostas por Schwartsburd e Labiak (2007), que categorizam as espécies em: Sul-Americanas (SA) - Aquelas com registro somente em países da América do Sul; Americanas (AM) - Ocorrem nas Américas do Sul, Central e do Norte; Atlânticas (AT) - Ocorrem na América, África e eventualmente em ilhas do Oceano Atlântico; Pacíficas (PA) - Ocorrem na América, Ásia e/ou Oceania; e Circum-Antárticas (CA) - Ocorrem na América, África, Ásia e/ou Oceania. Os registros de ocorrência das espécies nos países do continente americano e em outros continentes - necessárias para seu enquadramento nas categorias listadas acima - foram obtidos nas obras utilizadas na identificação das espécies.

Uma chave de identificação do tipo dicotômica e emparelhada numérica foi elaborada para as espécies registradas no Rancho Souza, com base na forma de vida e em características morfológicas de plantas maduras (apenas esporófitos). 
Research, Society and Development, v. 11, n. 2, e42611224890, 2022

(CC BY 4.0) | ISSN 2525-3409 | DOI: http://dx.doi.org/10.33448/rsd-v11i2.24890

Figura 2. Ambientes reconhecidos no Rancho Souza em Abaetetuba-PA, Brasil: A. Interior da mata, mostrando árvores de grande porte formando um dossel fechado. B. Clareira em área de terra firme. C. Sub-bosque em área de várzea. D. Margem da mata, em área de contato com roçado. E. Área de pasto (local aberto) com palmeiras de inajá. F. Remanescente florestal (ao fundo) envolto por uma área de roçado/pasto (em primeiro plano).
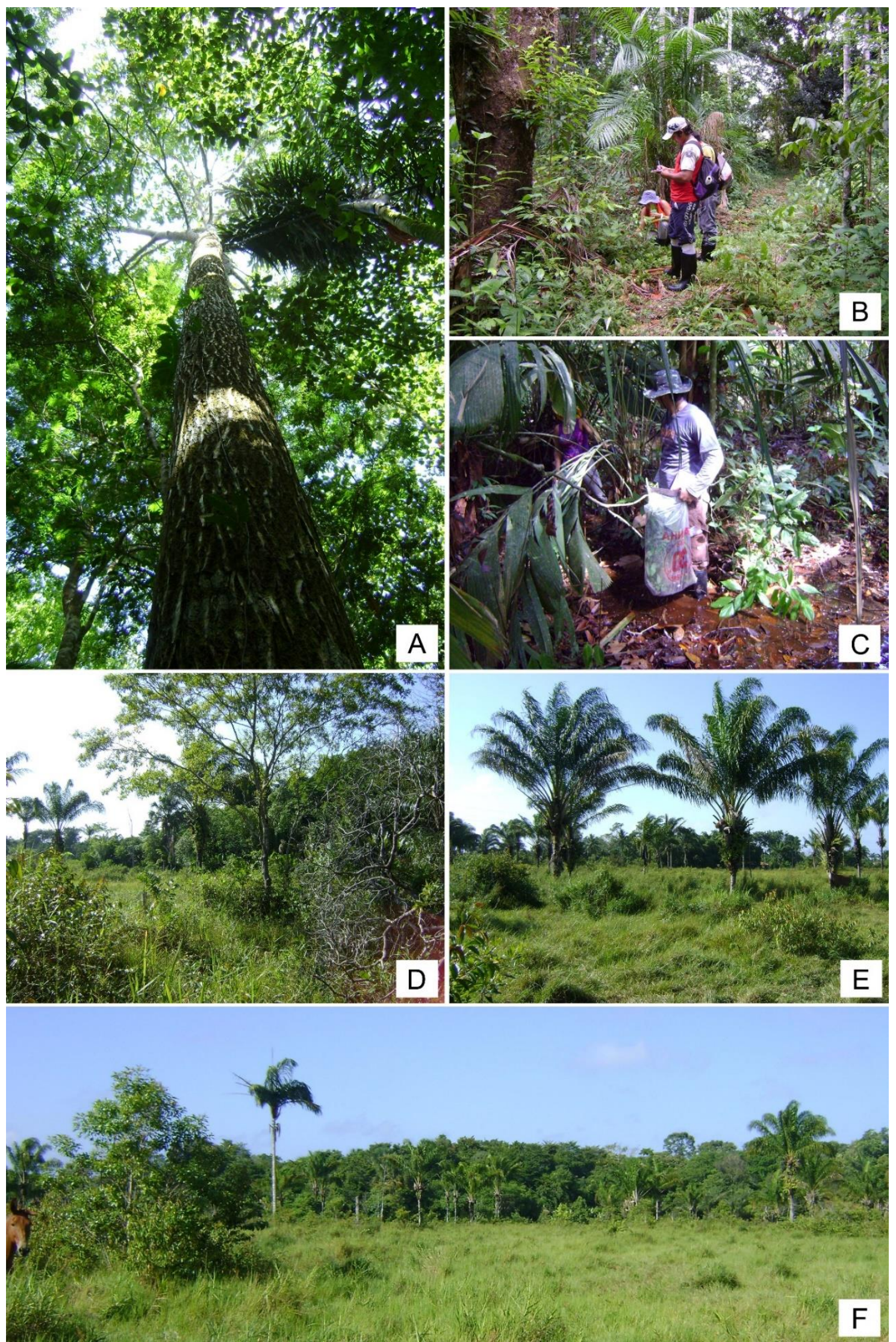

Fonte: Banco de Imagens do HIFPA (fotos de Jeferson Miranda Costa). 


\section{Resultados e Discussão}

No Rancho Souza foram registradas 31 espécies de plantas vasculares sem sementes, sendo uma licófita (Selaginella conduplicata Spring, Selaginellaceae, Figura 3A) e 30 samambaias, distribuídas em 22 gêneros e 14 famílias (Tabela 1). O total de espécies registradas na área representa 9,5\% da flora de samambaias e licófitas do Pará, composta por 326 espécies, segundo dados da Flora do Brasil 2020. Comparando com os resultados obtidos em um levantamento realizado em Barcarena, município limítrofe de Abaetetuba, a riqueza específica de samambaias e licófitas do Rancho Souza foi aproximadamente 25\% menor que a registrada na Ilha de Trambioca, para onde foram listadas 41 espécies por Teixeira et al. (2015). No entanto, com uma área 170 vezes menor que a da referida ilha (que tem 8.486 hectares), o Rancho Souza possui uma riqueza de espécies relativamente significativa.

As famílias mais representativas registradas na área foram Pteridaceae, com seis espécies, seguida de Hymenophyllaceae e Polypodiaceae, com quatro espécies cada (Tabela 1), resultado similar ao obtido na Ilha de Trambioca (Teixeira et al., 2015). Em levantamentos florísticos feitos em outras áreas próximas à Abaetetuba, como na região metropolitana de Belém (Costa \& Pietrobom, 2010) e na Floresta Nacional de Caxiuanã em Melgaço (Pietrobom et al., 2015), Dryopteridaceae também está entre as famílias de maior representatividade em função da riqueza específica dos gêneros Elaphoglossum Schott. ex J. Sm. e Cyclodium C. Presl. De fato, no Campo Experimental da Embrapa Amazônia Oriental em Moju, município vizinho de Abaetetuba, Dryopteridaceae está representada por três espécies de Cyclodium e seis espécies de Elaphoglossum (Maciel \& Pietrobom, 2010), das quais E. luridum (Fée) H. Christ é bastante comum e foi coletada por Teixeira et al. (2015) na Ilha de Trambioca em Barcarena. Entretanto, apenas uma espécie dessa família foi coletada no Rancho Souza (Polybotrya caudata Kunze, Figura 3D).

Os gêneros com maior riqueza específica no Rancho Souza foram Cyathea J. Sm. (Cyatheaceae) e Didymoglossum Desv. (Hymenophyllaceae), com três espécies cada (Tabela 1). Cyathea está entre os táxons de samambaias mais citados como indicadores ambientais (Della \& Falkenberg, 2019), uma vez que as espécies do gênero apresentam taxa de crescimento muito lenta e podem ser relacionadas ao estágio sucessional das florestas (Schmitt \& Windisch, 2012). Castello et al. (2017) demonstraram que samambaias arborescentes com caules desenvolvidos (acima 4,1 $\mathrm{m}$ de altura) podem ser usadas como indicadoras de vegetação bem conservada, já que tal característica é positivamente influenciada por fatores ambientais encontrados em estágios sucessionais avançados, como cobertura do dossel mais densa, maior umidade e menor exposição à luz. Os espécimes de Cyathea coletados no Racho Souza não possuíam caules desenvolvidos em comprimento (Figura 3C), diferente do observado por Costa et al. (2006) no Parque Ambiental de Belém, onde alguns indivíduos de Cyathea microdonta (Desv.) Domin alcançavam até cinco metros de altura. Já as espécies de Didymoglossum coletadas na área foram reconhecidas por Gehrig-Downie et al. (2012) como indicadoras de florestas de várzea, principalmente D. kapplerianum (J.W. Sturm) Ebihara \& Dubuisson (Figura 3E), o que foi corroborado no presente estudo.

Em relação ao hábito, 26 espécies são herbáceas, três são arborescentes (gênero Cyathea) e duas são trepadeiras (Tabela 1). Considerando as espécies arborescentes listadas em estudos feitos em regiões próximas à Abaetetuba (Costa \& Pietrobom, 2010; Pietrobom et al., 2015), apenas C. surinamensis (Miq.) Domin não foi encontrada no Rancho Souza. As duas espécies trepadeiras registradas na área contrastam em suas características: Salpichlaena hookeriana (Kuntze) Alston cresce comumente ao longo de igarapés no interior das matas ciliares e é pouco tolerante a alterações ambientais (Costa \& Pietrobom, 2007), enquanto Lygodium volubile Sw. (Figura 3H) ocorre principalmente em áreas de vegetação secundária, demonstrando bastante tolerância às perturbações (Travassos et al., 2014).

Quanto às formas de vida das samambaias e licófitas do Rancho Souza, foram registradas 17 espécies exclusivamente terrestres, 12 epífitas e duas hemiepífitas na maturidade (Tabela 1). Apenas Polybotrya caudata apresentou duas formas de vida dependendo do seu estágio de desenvolvimento, sendo terrestre quando jovem e se tornando hemiepífita na maturidade (Figura 
3D), o que já foi observado por Costa et al. (2006) no Parque Ambiental do Utinga e por Ferreira et al. (2009) na Ilha do Combu, ambos em Belém. Vale ressaltar que espécies epífitas comuns na região não foram registradas na área, tais como as polipodiáceas Microgramma lycopodioides (L.) Copel., Phlebodium decumanum (Willd.) J. Sm. e Pleopeltis desvauxii (Klotzsch) Salino, além da intrigante ausência do gênero Elaphoglossum, cujas espécies habitam preferencialmente formações primárias e são muito sensíveis a modificações das condições existentes (Brade, 2003). De fato, as espécies de Elaphoglossum registradas no Campo Experimental da Embrapa em Moju foram observadas apenas no interior das matas de terra firme e/ou igapó, geralmente ocorrendo nas margens de rios (Maciel \& Pietrobom, 2010), o que demonstra uma preferência das espécies locais por ambientes florestais mais preservados.

A maioria das espécies de samambaias e licófitas do Rancho Souza foram encontradas em ambientes associados ao remanescente florestal da área, registrando-se 24 espécies no interior da mata (sendo 14 exclusivas deste ambiente), $13 \mathrm{em}$ clareiras e quatro nas margens da mata (Tabela 1). Tal predominância de espécies no interior da mata está associada à dependência que a maioria das plantas vasculares sem sementes tem pelo abrigo e suporte oferecidos por outras plantas, sobretudo na fase gametofítica e durante o estabelecimento do esporófito jovem (Holttum, 1938). Apenas quatro espécies epífitas foram coletadas em locais abertos (Ananthacorus angustifolius (Sw.) Underw. \& Maxon, Campyloneurum phyllitidis (L.) C. Presl, Pleopeltis burchellii (Baker) Hickey \& Sprunt ex A.R. Sm., e Serpocaulon triseriale (Sw.) A.R. Sm.), três das quais crescendo sobre estipe de palmeiras de inajá em área de roçado (Figura 4C). A tolerância destas espécies epífitas a ambientes alterados foi registrada por Costa e Pietrobom (2007), que as observaram ocorrendo em áreas urbanas sobre árvores localizadas em propriedades particulares, praças e ao longo das vias públicas da Ilha de Mosqueiro em Belém.

Quanto ao padrão de distribuição geográfica das espécies ocorrentes no Rancho Souza, foram registradas 25 espécies americanas, quatro sulamericanas, uma pacífica e uma circum-antártica (Tabela 1). Segundo Tryon e Tryon (1982), das 3.250 espécies de plantas vasculares sem sementes ocorrentes nas Américas, 3.000 são exclusivas dos neotrópicos, o que justifica a identificação de apenas duas espécies, na área estudada, com distribuição que ultrapassa os limites do continente americano (Telmatoblechnum serrulatum (Rich.) Perrie, D.J. Ohlsen \& Brownsey, com distribuição pacífica, e Nephrolepis biserrata (Sw.) Schott, uma espécie circum-artártica). Nenhuma das espécies com distribuição sulamericana listadas neste estudo é endêmica da Amazônia ou mesmo restrita ao Brasil (Flora do Brasil 2020). Esse resultado corrobora com a constatação de Tryon e Conant (1975), segundo os quais a biogeografia da pteridoflora amazônica é marcada por um baixo endemismo (ou mesmo inexistente) em uma área muito grande. 
Figura 3. Espécies coletadas no Rancho Souza em Abaetetuba-PA, Brasil: A. Selaginella conduplicata Spring. B. Asplenium serratum L. C. Cyathea cyatheoides (Desv.) K.U. Kramer. D. Polybotrya caudata Kunze, hemiepífita. E. Didymoglossum kapplerianum (J.W. Sturm) Ebihara \& Dubuisson, crescendo entre os musgos. F. Lindsaea lancea (L.) Bedd. var. lancea. G. Lomariopsis prieuriana Fée. H. Lygodium volubile Sw.
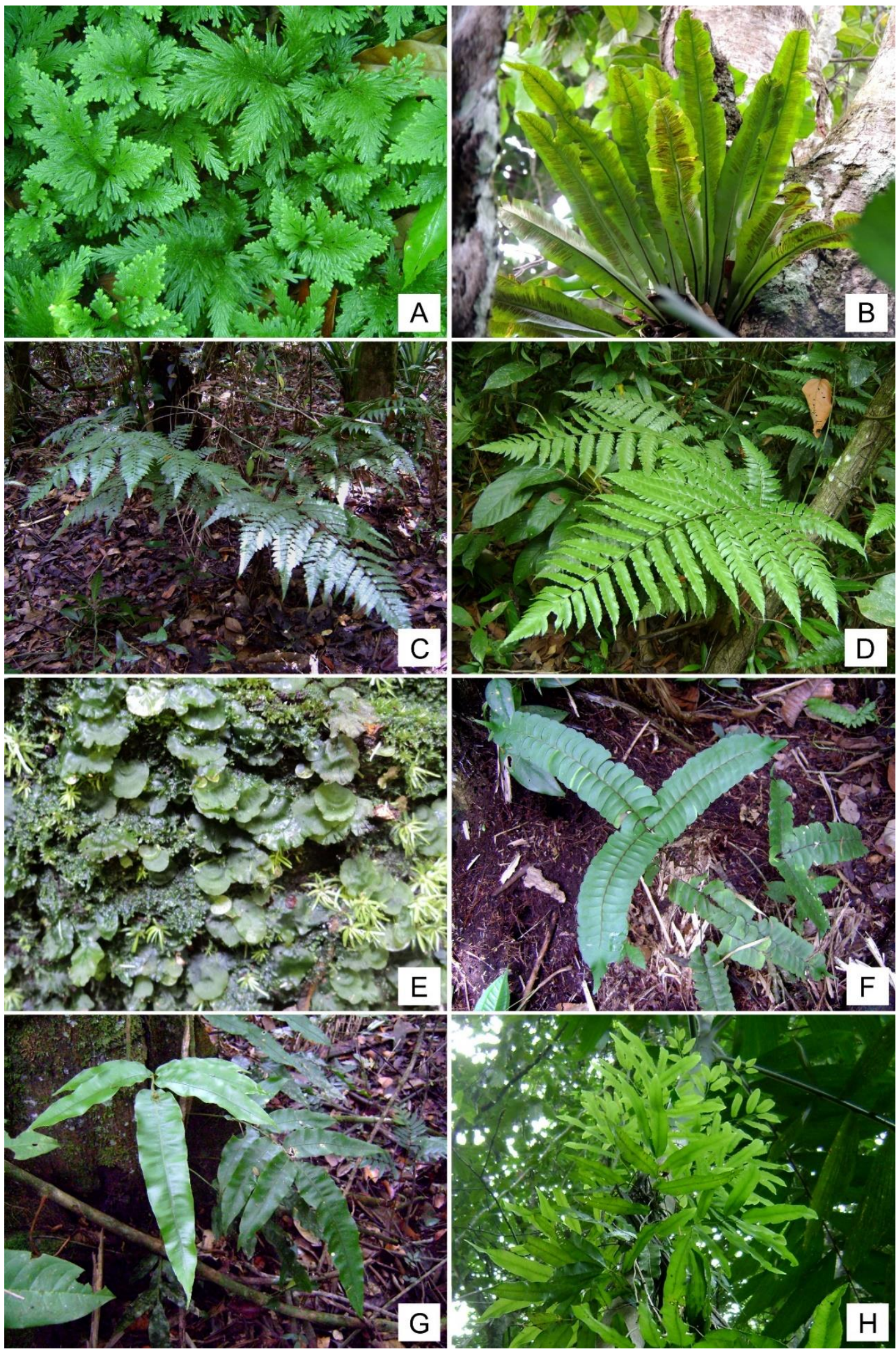

Fonte: Banco de Imagens do HIFPA (fotos de Jeferson Miranda Costa). 
Research, Society and Development, v. 11, n. 2, e42611224890, 2022

(CC BY 4.0) | ISSN 2525-3409 | DOI: http://dx.doi.org/10.33448/rsd-v11i2.24890

Figura 4. Espécies coletadas no Rancho Souza em Abaetetuba-PA, Brasil: A. Danaea simplicifolia Rudge. B. Danaea trifoliata Rchb. ex Kunze. C. Campyloneurum phyllitidis (L.) C. Presl. D. Microgramma reptans (Cav.) A.R. Sm. E. Adiantum latifolium Lam. F. Ananthacorus angustifolius (Sw.) Underw. \& Maxon. G. Triplophyllum funestum (Kunze) Holttum. H. Meniscium macrophyllum Kunze.
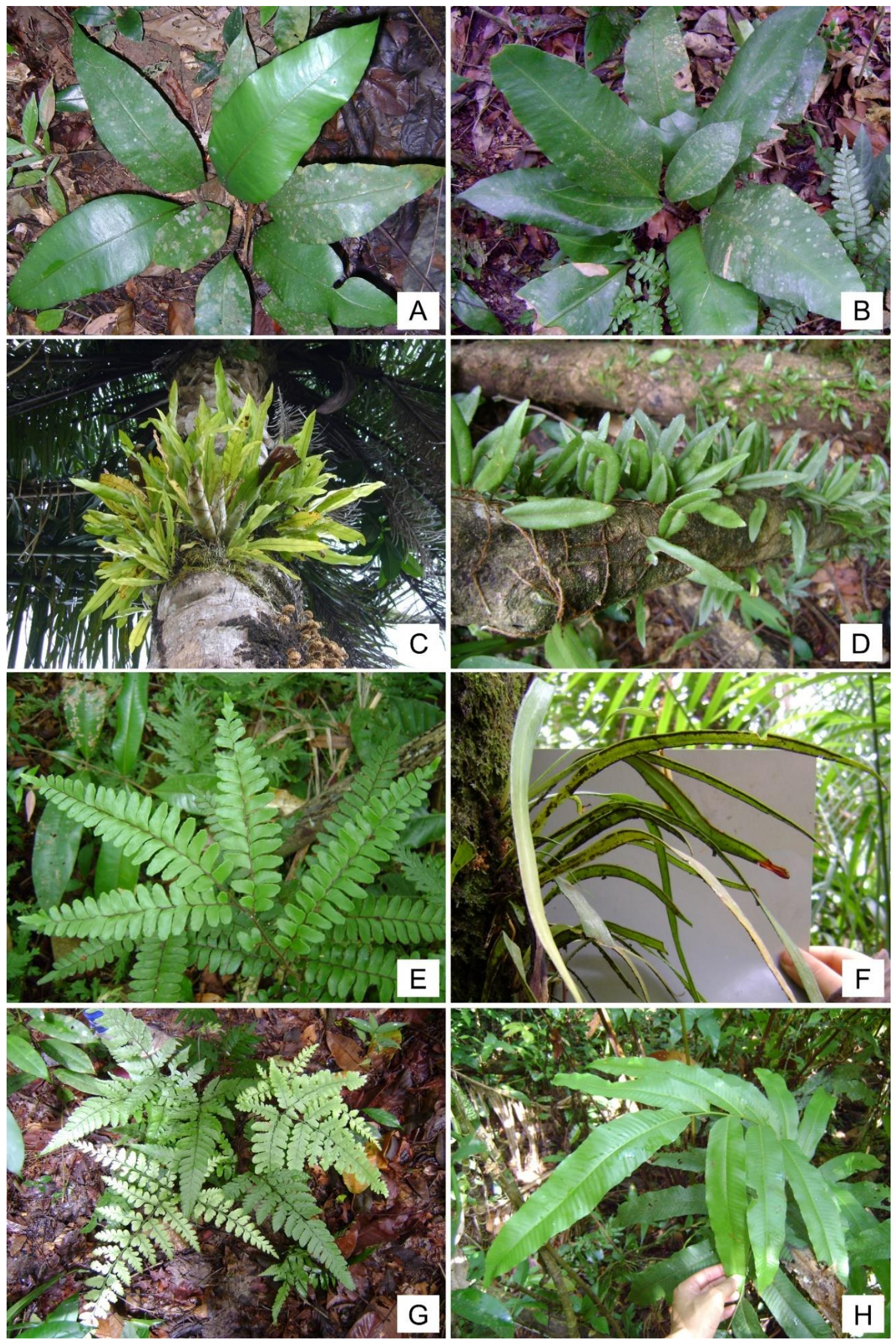

Fonte: Banco de Imagens do HIFPA (fotos de Jeferson Miranda Costa). 
Tabela 1. Samambaias e licófitas ocorrentes no Rancho Souza em Abaetetuba-PA, com suas respectivas informações sobre forma de vida, ambientes de ocorrência, padrões de distribuição e vouchers. Legenda: HA: hábitos; HE: herbáceo; TR: trepadeira; AR: arborescente; FV: formas de vida; TE: terrestre; EP: epífita; HE: hemiepífita; AO: ambientes de ocorrência; IM: interior da mata; CL: clareira; MM: margem da mata; LA: local aberto; PD: padrões de distribuição; AM: americana; AS: sul-americana; PA: pacífica; CA: circum-antártica.

\begin{tabular}{|c|c|c|c|c|c|}
\hline TÁXONS & HA & $\mathbf{F V}$ & AO & PD & VOUCHERS \\
\hline \multicolumn{6}{|l|}{ LICÓFITAS } \\
\hline \multicolumn{6}{|l|}{ Selaginellaceae } \\
\hline Selaginella conduplicata Spring & $\mathrm{HE}$ & $\mathrm{TE}$ & $\mathrm{IM} / \mathrm{CL}$ & SA & Bonneterre et al. 8,50 \\
\hline \multicolumn{6}{|l|}{ SAMAMBAIAS } \\
\hline \multicolumn{6}{|l|}{ Aspleniaceae } \\
\hline Asplenium serratum $\mathrm{L}$. & $\mathrm{HE}$ & EP & IM & $\mathrm{AM}$ & Bonneterre et al. 41,43 \\
\hline \multicolumn{6}{|l|}{ Blechnaceae } \\
\hline Salpichlaena hookeriana (Kuntze) Alston & TR & $\mathrm{TE}$ & IM & SA & Bonneterre et al. 40 \\
\hline $\begin{array}{l}\text { Telmatoblechnum serrulatum (Rich.) Perrie, D.J. } \\
\text { Ohlsen \& Brownsey }\end{array}$ & $\mathrm{HE}$ & $\mathrm{TE}$ & $\mathrm{MM} / \mathrm{CL}$ & PA & Bonneterre et al. 26 \\
\hline \multicolumn{6}{|l|}{ Cyatheaceae } \\
\hline Cyathea cyatheoides (Desv.) K.U. Kramer & AR & $\mathrm{TE}$ & IM & SA & Bonneterre et al. 67 \\
\hline Cyathea microdonta (Desv.) Domin & AR & $\mathrm{TE}$ & IM & $\mathrm{AM}$ & Bonneterre et al. 20 \\
\hline Cyathea pungens (Willd.) Domin & AR & TE & $\mathrm{IM} / \mathrm{CL}$ & $\mathrm{AM}$ & $\begin{array}{l}\text { Bonneterre et al. } 23,24,34 \mathrm{~A}, \\
\text { Costa \& Sena } 895\end{array}$ \\
\hline \multicolumn{6}{|l|}{ Dryopteridaceae } \\
\hline Polybotrya caudata Kunze & $\mathrm{HE}$ & $\mathrm{TE} / \mathrm{HE}$ & IM & $\mathrm{AM}$ & Bonneterre et al. 21, 22, 28 \\
\hline \multicolumn{6}{|l|}{ Hymenophyllaceae } \\
\hline $\begin{array}{l}\text { Didymoglossum kapplerianum (J.W. Sturm) Ebihara } \\
\text { \& Dubuisson }\end{array}$ & $\mathrm{HE}$ & EP & $\mathrm{IM} / \mathrm{CL}$ & $\mathrm{AM}$ & Bonneterre et al. 27, 31, 52, 53 \\
\hline Didymoglossum pinnatinervium (Jenman) Pic. Serm. & $\mathrm{HE}$ & EP & IM & $\mathrm{AM}$ & Bonneterre et al. 36B \\
\hline Didymoglossum punctatum (Poir.) Desv. & $\mathrm{HE}$ & EP & IM & $\mathrm{AM}$ & Bonneterre et al. 36A \\
\hline Trichomanes pinnatum Hedw. & $\mathrm{HE}$ & $\mathrm{TE}$ & $\mathrm{IM} / \mathrm{CL}$ & $\mathrm{AM}$ & Bonneterre et al. 15,57 \\
\hline \multicolumn{6}{|l|}{ Lindsaeaceae } \\
\hline Lindsaea lancea (L.) Bedd. var. lancea & $\mathrm{HE}$ & $\mathrm{TE}$ & IM & $\mathrm{AM}$ & Bonneterre et al. 29, 51 \\
\hline \multicolumn{6}{|l|}{ Lomariopsidaceae } \\
\hline Lomariopsis prieuriana Fée & $\mathrm{HE}$ & $\mathrm{HE}$ & IM & $\mathrm{AM}$ & Bonneterre et al. 13, 60, 65 \\
\hline \multicolumn{6}{|l|}{ Lygodiaceae } \\
\hline Lygodium volubile $\mathrm{Sw}$. & TR & $\mathrm{TE}$ & $\mathrm{IM}$ & $\mathrm{AM}$ & Bonneterre et al. 39 \\
\hline
\end{tabular}


Research, Society and Development, v. 11, n. 2, e42611224890, 2022

(CC BY 4.0) | ISSN 2525-3409 | DOI: http://dx.doi.org/10.33448/rsd-v11i2.24890

\begin{tabular}{|c|c|c|c|c|c|}
\hline Danaea simplicifolia Rudge & $\mathrm{HE}$ & $\mathrm{TE}$ & $\mathrm{IM}$ & $\mathrm{AM}$ & Bonneterre et al. 55, 64 \\
\hline Danaea trifoliata Rchb. ex Kunze & $\mathrm{HE}$ & $\mathrm{TE}$ & $\mathrm{IM}$ & $\mathrm{AM}$ & Bonneterre et al. $17,38,45 \mathrm{~B}$ \\
\hline \multicolumn{6}{|l|}{ Nephrolepidaceae } \\
\hline Nephrolepis biserrata (Sw.) Schott & $\mathrm{HE}$ & TE & IM/CL & $\mathrm{CA}$ & Bonneterre et al. $45 \mathrm{~A}$ \\
\hline \multicolumn{6}{|l|}{ Polypodiaceae } \\
\hline Campyloneurum phyllitidis (L.) C. Presl & $\mathrm{HE}$ & $\mathrm{EP}$ & IM/LA & $\mathrm{AM}$ & Bonneterre et al. $32,47,71$ \\
\hline Microgramma reptans (Cav.) A.R. Sm. & $\mathrm{HE}$ & $\mathrm{EP}$ & $\mathrm{MM} / \mathrm{CL}$ & $\mathrm{AM}$ & Bonneterre et al. 10, 33, 66 \\
\hline $\begin{array}{l}\text { Pleopeltis burchellii (Baker) Hickey \& Sprunt ex A.R. } \\
\text { Sm. }\end{array}$ & $\mathrm{HE}$ & $\mathrm{EP}$ & LA & SA & Bonneterre et al. 48 \\
\hline Serpocaulon triseriale (Sw.) A.R. Sm. & $\mathrm{HE}$ & $\mathrm{EP}$ & MM/LA & $\mathrm{AM}$ & $\begin{array}{l}\text { Bonneterre et al. } 46 \text {, Costa \& Sena } \\
897\end{array}$ \\
\hline \multicolumn{6}{|l|}{ Pteridaceae } \\
\hline Adiantum latifolium Lam. & $\mathrm{HE}$ & $\mathrm{TE}$ & IM/CL & $\mathrm{AM}$ & $\begin{array}{l}\text { Bonneterre et al. } 9,11,14,16,18, \\
19,56,61,62\end{array}$ \\
\hline Adiantum petiolatum Desv. & $\mathrm{HE}$ & $\mathrm{TE}$ & $\mathrm{IM} / \mathrm{CL}$ & $\mathrm{AM}$ & Bonneterre et al. 12,68 \\
\hline Ananthacorus angustifolius (Sw.) Underw. \& Maxon & $\mathrm{HE}$ & $\mathrm{EP}$ & MM/LA & $\mathrm{AM}$ & $\begin{array}{l}\text { Bonneterre et al. } 72 \text {, Costa \& Sena } \\
896\end{array}$ \\
\hline Polytaenium citrifolium (L.) Schuettp. & $\mathrm{HE}$ & $\mathrm{EP}$ & $\mathrm{CL}$ & $\mathrm{AM}$ & Bonneterre et al. 30 \\
\hline Polytaenium guayanense (Hieron.) Alston & $\mathrm{HE}$ & $\mathrm{EP}$ & $\mathrm{IM}$ & $\mathrm{AM}$ & Bonneterre et al. 37,58 \\
\hline Vittaria lineata $(\mathrm{L}.) \mathrm{Sm}$. & $\mathrm{HE}$ & $\mathrm{EP}$ & $\mathrm{IM}$ & $\mathrm{AM}$ & Bonneterre et al. 42 \\
\hline \multicolumn{6}{|l|}{ Tectariaceae } \\
\hline Triplophyllum funestum (Kunze) Holttum & $\mathrm{HE}$ & $\mathrm{TE}$ & $\mathrm{IM} / \mathrm{CL}$ & $\mathrm{AM}$ & Bonneterre et al. 34B, 35, 54, 59 \\
\hline \multicolumn{6}{|l|}{ Thelypteridaceae } \\
\hline Meniscium macrophyllum Kunze & $\mathrm{HE}$ & $\mathrm{TE}$ & $\mathrm{IM} / \mathrm{CL}$ & $\mathrm{AM}$ & Bonneterre et al. 25, 69, 70 \\
\hline Meniscium serratum Cav. & $\mathrm{HE}$ & TE & CL & $\mathrm{AM}$ & Bonneterre et al. 49 \\
\hline
\end{tabular}

Fonte: Autores.

Chave de identificação para as espécies de samambaias e licófitas do Rancho Souza, Abaetetuba-PA, Brasil

1. Folhas do tipo microfilo Selaginella conduplicata

1'. Folhas do tipo megáfilo

.2 (Samambaias)

2. Frondes com apenas uma camada de células no tecido laminar.................................................. 3

2'. Frondes com várias camadas de células no tecido laminar.

3. Caules curto-reptantes a decumbentes ou eretos, com raízes; lâminas $>10 \mathrm{~cm}$ compr., 1 pinadas Trichomanes pinnatum

3'. Caules longo-reptantes, sem raízes; lâminas até $2 \mathrm{~cm}$ compr., simples, inteiras ou lobadas..... 4

4. Lâminas com margem glabra; falsas vênulas submarginais presentes. Didymoglossum kapplerianum

4'. Lâminas com margem pilosa; falsas vênulas submarginais ausentes. .5 
5. Margem da lâmina com tricomas simples ou usualmente geminados; costa percorrente; venação pinada; indúsios sem lábios distintos, com margem não escurecida...

Didymoglossum pinnatinervium

5'. Margem da lâmina com tricomas estrelados; costa não percorrente; venação flabelada; indúsios com lábios distintos e margem escurecida...

Didymoglossum punctatum

6. Plantas epífitas 7

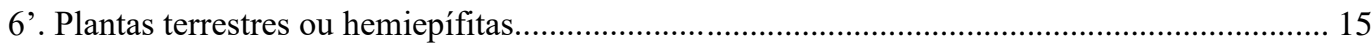

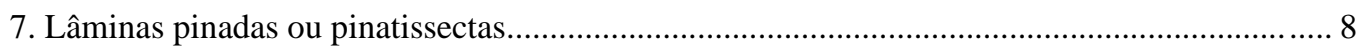

7'. Lâminas simples. 9

8. Lâminas escamosas em ambas as superfícies; venas não visíveis. Pleopeltis burchellii

8'. Lâminas glabras em ambas as superfícies; venas visíveis Serpocaulon triseriale

9. Venação livre; indúsios presentes. .Asplenium serratum

9'. Venação areolada; indúsios ausentes .10

10. Lâminas linear-elípticas a/ou lineares; soros lineares, submarginais. 11

10'. Lâminas ovaladas, lanceoladas, oblanceoladas ou oblongo-lanceoladas; soros circulares, subacrosticóides ou lineares, dispostos entre a costa e a margem (não submarginais).

11. Lâminas 0,2-0,3 cm larg., lineares, com uma fileira de aréolas alongadas entre a costa e a margem. Vittaria lineata

11'. Lâminas 0,6-1,2 cm larg., linear-elípticas a lineares, com 2-4 fileiras de aréolas alongadas entre a costa e a margem. .Ananthacorus angustifolius

12. Frondes dimórficas; lâminas escamosas Microgramma reptans

12'. Frondes monomórficas; lâminas glabras. 13

13. Aréolas com vênulas livres inclusas; soros circulares Campyloneurum phyllitidis

13'. Aréolas sem vênulas livres inclusas; soros subacrosticóides ou lineares. 14

14. Caules curto-reptantes; soros lineares em arranjo reticulado, esporângios somente sobre as venas. Polytaenium guayanense

14'. Caules longo-reptantes; soros subacrosticóides, esporângios dispostos individualmente ou agrupados sobre e entre as venas Polytaenium citrifolium

15. Plantas trepadeiras; frondes com raque volúvel 16

15'. Plantas não trepadeiras; frondes com raque não volúvel. 17

16. Lâminas 3-pinadas; esporângios individualmente protegidos pela margem da lâmina modificada...

..Lygodium volubile

16'. Lâminas 2-pinadas; esporângios agrupados em soros lineares, paralelos e adjacentes à cóstula Salpichlaena hookeriana

17. Plantas hemiepífitas na maturidade. . .18

17'. Plantas terrestres. 19

18. Lâminas estéreis 1-pinadas, com segmento apical livre; pinas articuladas com a raque.

.Lomariopsis prieuriana 18'. Lâminas estéreis 1-2-pinado-pinatífidas, com ápice pinatífido; pinas não articuladas com a raque. Polybotrya caudata

19. Pecíolos com um ou dois nós; soros com esporângios reunidos em sinângios. 20

19’. Pecíolos sem nós; soros com esporângios livres 21

20. Pecíolos com um nó; lâminas simples Danaea simplicifolia 
20'. Pecíolos com dois nós; lâminas simples ou 1-pinadas (com 1 par de pinas laterais ou somente uma pina lateral).

Danaea trifoliata

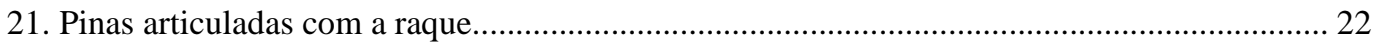

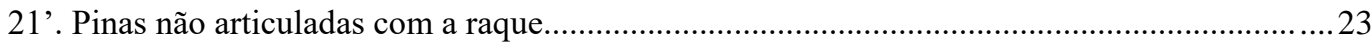

22. Pinas com margens não cartilaginosas; soros circulares; indúsios reniformes. .Nephrolepis biserrata

22'. Pinas com margens cartilaginosas; soros lineares; indúsios lineares. Telmatoblechnum serrulatum

23'. Lâminas 2-pinado-pinatífidas, com ápice pinatífido. 24

23. Lâminas 1 a 2-pinadas, com segmento apical livre............................................................... 27

24. Plantas herbáceas; caule reptante a decumbente. Triplophyllum funestum

24'. Plantas arborescentes; caule ereto .25

25. Pecíolos com escamas concolores na base; indúsios presentes Cyathea cyatheoides

25’. Pecíolos com escamas bicolores na base; indúsios ausentes .26

26. Raque com espinhos até próximo ao ápice; raquíolas das pinas proximais com espinhos em pelo menos até $2 / 3$ de sua extensão Cyathea microdonta

26'. Raque inerme ou com espinhos somente próximos ao pecíolo; raquíolas inermes Cyathea pungens

27. Pecíolos e raques castanho-escuros a negros. 28

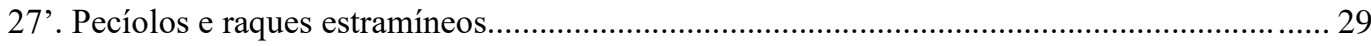

28. Caules curto-reptantes; lâminas 1-2-pinadas. Adiantum petiolatum

28'. Caules longo-reptantes; lâminas 2-pinadas Adiantum latifolium

29. Lâminas 2-pinadas; venação livre; soros submarginais Lindsaea lancea var. lancea

29'. Lâminas 1-pinadas; venação areolada; soros dispostos entre a costa e a margem (não submarginais) 30

30. Pinas com margem uncinado-serreada Meniscium serratum

30'. Pinas com margem inteira a irregularmente crenulada ou ondulada Meniscium macrophyllum

\section{Considerações Finais}

A flora de samambaias e licófitas registrada no Rancho Souza apresentou uma riqueza significativa ao se considerar o tamanho da área amostrada e talvez pudesse ter sido ainda maior, caso tivessem sido empregadas técnicas de coleta direcionadas a epífitas de estratos superiores da mata (dossel).

Como esperado, foi nos ambientes florestais do Rancho Souza que se registrou a maior diversidade de espécies, principalmente no interior das matas de terra firme e várzea, demonstrando a importância da preservação dos remanescentes florestais por menores que sejam, sobretudo aqueles que incluem matas ciliares.

A ausência de alguns táxons (gênero Elaphoglossum) ou mesmo a forma como alguns se apresentaram (espécies de Cyathea com caules pouco desenvolvidos) podem ser indicativos de que o remanescente florestal da área esteja em processo de regeneração relativamente recente.

No entanto, embora imersa em uma matriz de ambientes degradados e sujeita aos efeitos de borda e de redução de área, notou-se que a área de mata mantida em regeneração natural no Rancho Souza oferece condições favoráveis ao estabelecimento de algumas espécies típicas de ambientes conservados.

De fato, é notório a forte pressão antrópica a que esta e outras áreas de floresta estão submetidas no município de Abaetetuba, seja pela expansão de áreas de ocupação humana, seja pela ampliação de áreas de pasto, cultivos agrícolas entre outros empreendimentos degradadores, como pontos de extração de areia. 
Nesse sentido, espera-se que este trabalho alerte para a importância da preservação dos remanescentes florestais de Abaetetuba e contribua com os próximos levantamentos florísticos de samambaias e licófitas que precisam ser feitos no município, principalmente em áreas sob forte e crescente pressão de degradação, como (1) a sub-bacia do rio Abaeté, que inclui parte da zona urbana da sede municipal e suas principais vias de acesso; (2) a região do Distrito de Beja, mais precisamente as sub-bacias dos rios Arienga e Arapiranga de Beja por onde cruzará parte do trecho 01 da Ferrovia Paraense, cujo objetivo será o transporte de minério e produtos do agronegócio; e (3) a região de Urubuéua e Ilha do Capim na "região das ilhas", pela instalação portuária do Terminal de Abaetetuba, que visará o escoamento de grãos para o mercado externo por via fluvial.

\section{Agradecimentos}

Ao professor Welbert José e Silva de Souza por autorizar e incentivar as coletas no Rancho Souza, propriedade pertencente à sua família; e a Raimundo dos Santos Sena, "Seu Barriga", por ter atuado como guia nas coletas.

\section{Referências}

Arcand, N. N. \& Ranker, T. A. (2008). Conservation biology. In: Ranker, A. T.; Haufler, C. H. (Eds.). Biology and Evolution of Ferns and Lycophytes. Cambridge University Press. Pp. 257-283.

Berry, P. E., Holst, B. K. \& Yatskievych, K. (1995). Pteridophytes, Spermatophytes: Acanthaceae-Araceae. In: Steyermark, J. S., Berry, P. E. \& Holst, B. K. (Eds.). Flora of the Venezuelan Guayana. Vol. 2. Missouri Botanical Garden, Timber Press. Pp. 1-706.

Boer, J. G. W. (1962). The New World Species of Trichomanes Sect. Didymoglossum and Microgonium. Acta Botanica Neerlandica 11: 277-330.

Brade, A. C. (2003). O gênero Elaphoglossum (Pteridophyta) no Brasil. Obra póstuma. Unisinos, Universidade do Vale do Rio dos Sinos, São Leopoldo. 204p. Cárdenas, G. G., Lehtonen, S. \& Tuomisto, H. (2019). Taxonomy and evolutionary history of the neotropical fern genus Salpichlaena (Blechnaceae). Blumea 64: $1-22$.

Castello, A. C. D., Coelho, S. \& Cardoso-Leite, E. (2017). Lianas, tree ferns and understory species: indicators of conservation status in the Brazilian Atlantic Rainforest remnants, southeastern Brazil. Brazilian Journal of Biology 77(2): 213-226.

Coelho, A. S., Toledo, P. M., Vieira, I. C. G., Canto, O., Adami, M., Gomes, A. R. \& Narvaes, I. S. (2018). Impactos das mudanças de uso da terra nas áreas prioritárias para conservação da biodiversidade no nordeste do estado do Pará, Brasil. Boletim do Museu Paraense Emílio Goeldi, Ciências Naturais 13(1): 107120.

Cordeiro, I. M. C. C., Arbage, M. J. C. \& Schwartz, G. (2017). Nordeste do Pará: configuração atual e aspectos identitários. In: Cordeiro, I. M. C. C., RangelVasconcelos, L. G. T., Schwartz, G. \& Oliveira, F. A. (Org.). Nordeste Paraense: panorama geral e uso sustentável das florestas secundárias. EDUFRA, Belém, Pará. Pp. 19-58.

Costa, J. M. \& Pietrobom, M. R. (2007). Pteridófitas (Lycophyta e Monilophyta) da Ilha de Mosqueiro, município de Belém, estado do Pará, Brasil. Boletim do Museu Paraense Emílio Goeldi, Ciências Naturais 2: 45-55.

Costa, J. M. \& Pietrobom, M. R. (2010). Samambaias e licófitas do Parque Estadual do Gunma, município de Santa Bárbara do Pará, estado do Pará, Brasil. Rodriguésia 61: 223-232.

Costa, J. M., Souza, M. G. C. \& Pietrobom, M. R. (2006). Levantamento florístico das pteridófitas (Lycophyta e Monilophyta) do Parque Ambiental de Belém (Belém, Pará, Brasil). Revista de Biologia Neotropical 3: 4-12.

Christenhusz, M. J. M. (2010). Danaea (Marattiaceae) revisited: biodiversity, a new classification and ten new species of a neotropical fern genus. Botanical Journal of the Linnean Society 163: 360-385.

Della, A. P. \& Falkenberg, D. B. (2019). Pteridophytes as ecological indicators: an overview. Hoehnea 46: e522018. http://dx.doi.org/10.1590/2236-8906$52 / 2018$.

FAPESPA - Fundação Amazônia de Amparo a Estudos e Pesquisas do Pará (2015). Estatísticas Municipais Paraenses: Abaetetuba. Diretoria de Estatística e de Tecnologia e Gestão da Informação, Belém. 52p.

Fearnside, P. M. (2020). Desmatamento na Amazônia brasileira: História, índices e consequências. In: Fearnside, P. M. (Ed.). Destruição e Conservação da Floresta Amazônica, Vol. 1. Editora do INPA, Manaus, Amazonas. Pp. 7-19.

Fernandes, I. (2003). Taxonomia dos representantes de Cyatheaceae do nordeste oriental do Brasil. Pesquisas, Botânica 4: 1-54.

Fernandes, R. S. \& Salino, A. (2020). Taxonomic revision of Meniscium Schreber (Thelypteridaceae: Polypodiopsida). Phytotaxa 463(1): 001-127.

Ferreira, L. S. L., Costa, J. M. \& Pietrobom, M. R. (2009). As pteridófitas. In: Jardim, M. A. G. (Org.). Diversidad e biológica das áreas de proteção ambiental Ilhas do Combu e Algodoal-Maiandeua, Pará, Brasil. MPEG/MCT/CNPq, Belém, Pará. Pp. 13-40. 
Research, Society and Development, v. 11, n. 2, e42611224890, 2022

(CC BY 4.0) | ISSN 2525-3409 | DOI: http://dx.doi.org/10.33448/rsd-v11i2.24890

FLORA DO BRASIL 2020. Jardim Botânico do Rio de Janeiro. <http://floradobrasil.jbrj.gov.br/>. Acesso em: 01 dez. 2021.

Gehrig-Downie, C., Marquardt, J., Obregón, A., Bendix, J. \& Gradstein, S. R. (2012). Diversity and vertical distribution of filmy ferns as a tool for identifying the novel forest type "tropical lowland cloud forest". Ecotropica 18: 35-44.

Holttum, R. E. (1938). The ecology of tropical pteridophytes. In: Verdoom, F. R. (Ed.). Manual of Pteridology. Martinus Nijhoff, The Hague. Pp. $420-450$.

INPE (2020). Projeto de Monitoramento do Desmatamento na Amazônia Legal por Satélite (PRODES). <http://www.dpi.inpe.br/prodesdigital/prodesmunicipal.php>. Acesso em: 29 dez. 2021.

Maciel, S. \& Pietrobom, M. R. (2010). Dryopteridaceae e Lomariopsidaceae (Polypodiopsida) do Campo Experimental da Embrapa Amazônia Oriental, Moju, Pará, Brasil. Rodriguésia 61(3): 405-414.

Mickel, J. T. \& Smith, A. R. (2004). The Pteridophytes of Mexico. Memoirs of the New York Botanical Garden 88: 1-1070.

Moran, R. C. (1987). Monograph of the Neotropical Fern Genus Polybotrya (Dryopteridaceae). Illinois Natural History Survey Bulletin 34(1-6): 1-138.

Moran, R. C. (2000). Monograph of the neotropical species of Lomariopsis (Lomariopsidaceae). Brittonia 52: 55-111.

Moran, R. C. \& Riba, R. (1995). Psilotaceae a Salviniaceae. In: Davidse, G., Sousa, M. \& Knapp, S. (Ed.). Flora mesoamericana. Vol. 1. Universidad Nacional Autónoma de México, Ciudad de México. Pp. 1-470.

Moran, R. C. \& Smith, A. R. (2001). Phytogeographic relationships between neotropical and African-Madagascar pteridophytes. Brittonia 53(2): 304-351.

Mori, S. A., Silva, L. A. M. \& Lisboa, G. (1989). Manual de manejo do herbário fanerogâmico. (2a ed.), Centro de Pesquisas do Cacau, Ilheús.

Parris, B. S. (2001). Circum-Antarctic continental distribution patterns in pteridophyte species. Brittonia 53(2): 270-283.

Pietrobom, M. R., Medeiro, P. S., Fonseca, M. S. S., Maciel, S., Souza, M. G. C. \& Costa, J. M. (2015). Contribuição à preservação da Floresta Nacional de Caxiuanã, Pará, Brasil: licófitas e samambaias. Pesquisas, Botânica 68: 349-361.

PPG I (2016). A community-derived classification for extant lycophytes and ferns. Journal of Systematics and Evolution 54: 563-603.

Prado, J. \& Moran, R. C. (2008). Revision of the neotropical species of Triplophyllum (Tectariaceae). Brittonia 60(2): 103-130.

Schmitt, J. L. \& Windisch, P. G. (2012). Caudex growth and phenology of Cyathea atrovirens (Langsd. \& Fisch.) Domin (Cyatheaceae) in secondary forest, southern Brazil. Brazilian Journal of Biology 72(2): 397-405.

Schwartsburd, P. B. \& Labiak, P. H. (2007). Pteridófitas do Parque Estadual de Vila Velha, Ponta Grossa, Paraná, Brasil. Hoehnea 34(2): 159-209.

Teixeira, G., Maciel, S. \& Pietrobom, M. R. (2015). Potencial utilitário de licófitas e samambaias: aplicabilidade ao contexto amazônico. Biota Amazônia 5(1): 68-73.

Travassos, C. C., Jardim, M. A. G. \& Maciel, S. (2014). Florística e ecologia de samambaias e licófitas como indicadores de conservação ambiental. Biota Amazônia 4(4): 40-44.

Tryon, R. M. \& Conant, D. S. (1975). The ferns of Brazilian Amazonia. Acta Amazonica 5: 23-24.

Tryon, R. M. \& Tryon, A. F. (1982). Ferns and allied plants, with special reference to Tropical America. Springer-Verlag, New York, Heidelberg, Berlin. 857p.

Vieira, I. C. G., Silva, J. M. C. \& Toledo, P. M. (2005). Estratégias para evitar a perda de biodiversidade na Amazônia. Estudos Avançados 19(54): 153-164.

Weigand, A. \& Lehnert, M. (2016). The scaly tree ferns (Cyatheaceae-Polypodiopsida) of Brazil. Acta Botanica Brasilica 30(3): 336-350.

Zuquim, G., Costa, F. R. C., Prado, J. \& Tuomisto, H. (2008). Guia de samambaias e licófitas da REBIO Uatumã, Amazônia Central. INPA, Manaus. 316p. 